\title{
Correlates of maternal diet during pregnancy
}

\author{
Hanke $\mathrm{W}^{1}$, Kaluzny $\mathrm{P}^{1}$, Wesolowska $E^{1}$, Jankowska $\mathrm{A}^{1}$, Trafalska $\mathrm{E}^{2}$, Calamandrei $\mathrm{G}^{3}$, Polanska $\mathrm{K}^{1}$ \\ ${ }^{1}$ Department of Environmental Epidemiology, Nofer Institute of Occupational Medicine, Lodz, Poland \\ ${ }^{2}$ Department of Hygiene and Epidemiology, Medical University of Lodz, Poland \\ ${ }^{3}$ Center for Behavioral Sciences and Mental Health, National Institute of Health, Rome, Italy
}

Introduction

Conceptual and methodological limitations of a single or a few nutrients or foods:

1. The 'single nutrient' approach may be inadequate for taking into account complicated interactions among nutrients

2. The high level of intercorrelation among some nutrients makes it difficult to examine their separate effect

3. The effect of a single nutrient may be too small to detect, but the cumulative effects of multiple nutrients included in a dietary pattern may be sufficiently large to be detectable

4. The analyses based on a large number of nutrients or food items may produce statistically significant associations simply by chance

5. Because nutrient intakes are commonly associated with certain dietary patterns, 'single nutrient' analysis may potentially be confounded by the effect of dietary patterns

Looking at overall diet quality, through dietary patterns, is believed to represent a valid and meaningful measure of overall nutrient intake and is a promising approach when aiming to study diet related associations.

The aim of the study is to evaluate the correlates of maternal diet during pregnancy.

Materials and methods

Study design - Polish Mother and Child Cohort Study (REPRO_PL)

Study population - 1306 pregnant women (up to 12 weeks of single pregnancy,

no assisted conception, no pregnancy complications and no chronic diseases Maternal diet during pregnancy - Food Frequency Questionnaire (FFQ) in 20-24 weeks of pregnancy
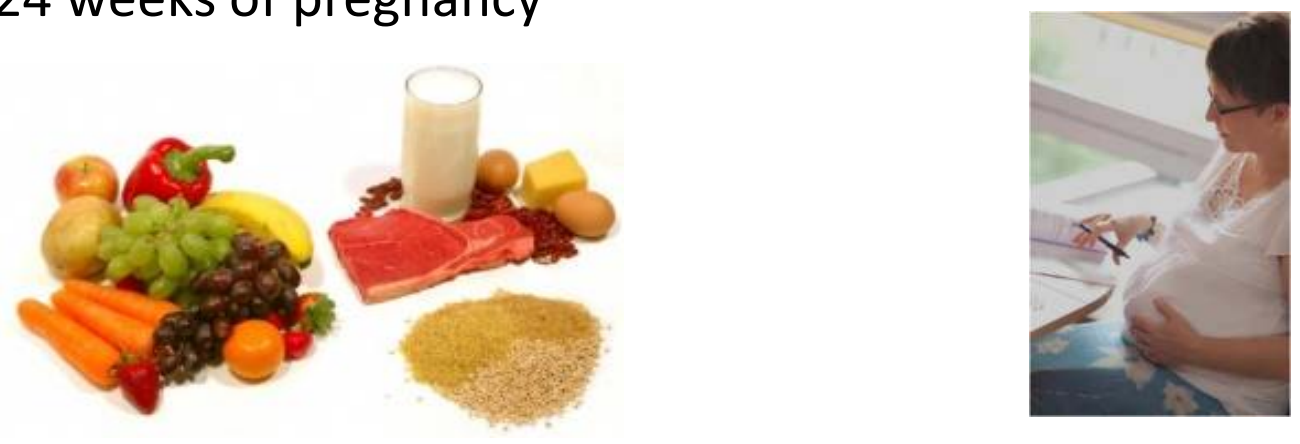

In FFQ the women were asked to report how often, on average, they consumed specified amounts of each food. The selected frequency category for each food item was converted to daily intake. Based on factor analysis two dietary patterns were identified.
- Red meat
- Processed meat
- Butter
- Potatoes
- Refined grains
- High-fat dairy
- Sweets

Unhealthily dietary pattern
Healthy dietary pattern

- Vegetables

- Fruits

- Legumes

- Whole grains

- Low-fat dairy

- Poultry

- Fish/seafood
Factor loadings for foods loaded on the two major dietary patterns identified from the FFQ

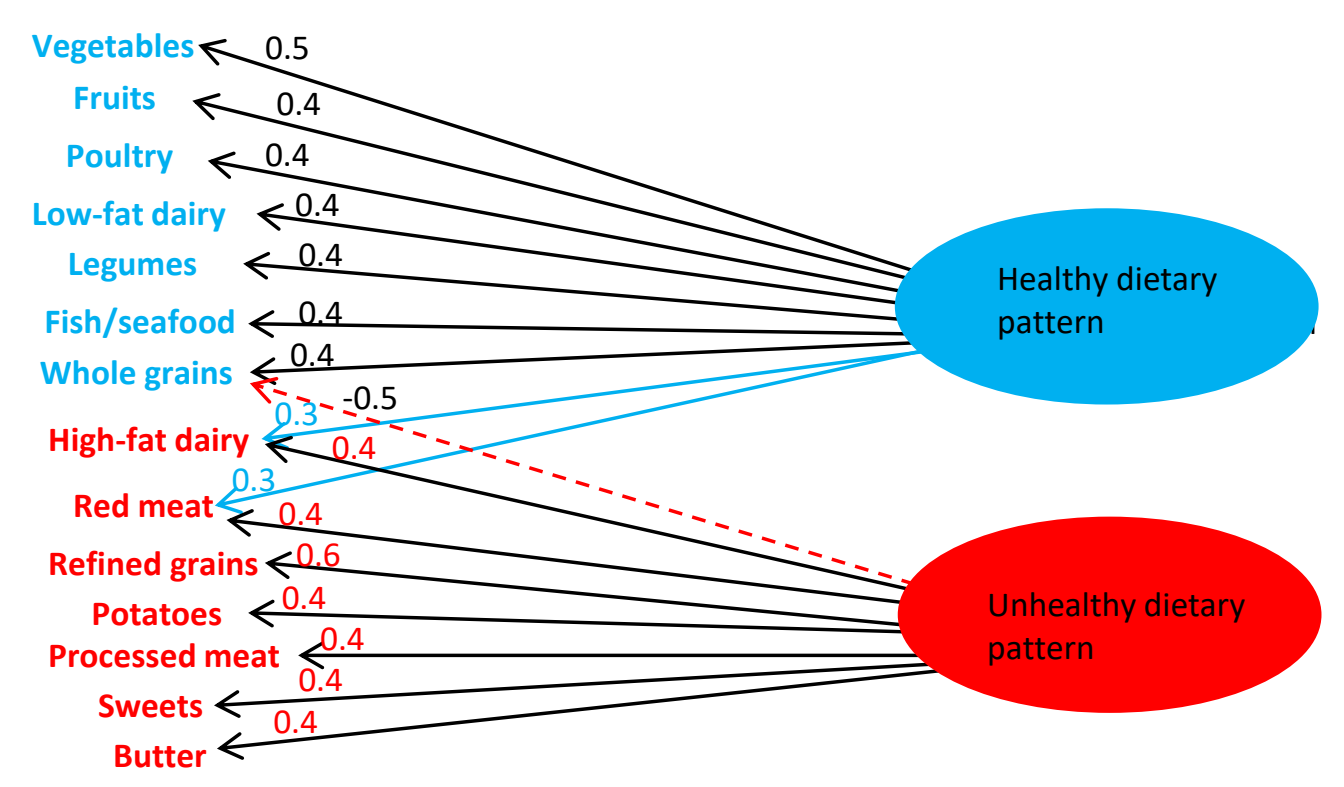

Results

Correlates of maternal diet during pregnancy - multivariate analysis

\begin{tabular}{|c|c|c|c|c|c|}
\hline \multicolumn{2}{|c|}{ Variables } & \multirow{2}{*}{$\begin{array}{c}\text { Unhealthy } \\
\text { dietary } \\
\text { pattern } \\
{[-4.25,-1.37]} \\
436(33.4)\end{array}$} & \multirow{2}{*}{$\begin{array}{c}\text { Mixed } \\
(-1.37,-0.44] \\
440(33.7)\end{array}$} & \multirow{2}{*}{\begin{tabular}{|c|}
$\begin{array}{c}\text { Healthy dietary } \\
\text { pattern } \\
(-0.44,4.42]\end{array}$ \\
$430(32.9)$
\end{tabular}} & \multirow[t]{2}{*}{$\mathbf{p}$} \\
\hline & & & & & \\
\hline Maternal age & $\leq 30$ & $296(35.4)$ & $279(33.3)$ & 262 (31.3) & 0.05 \\
\hline [years] & $>30$ & 130 (29.1) & $153(34.3)$ & $163(36.5)$ & \\
\hline \multirow[t]{2}{*}{ Marital status } & Married & 333 (31.9) & 351 (33.6) & 361 (34.5) & 0.03 \\
\hline & Single & $97(38.8)$ & $87(34.8)$ & $66(26.4)$ & \\
\hline \multirow{3}{*}{$\begin{array}{c}\text { Maternal education } \\
\text { [years of } \\
\text { education }\end{array}$} & $>12$ & 261 (28.9) & $303(33.5)$ & 340 (37.6) & $<0.001$ \\
\hline & $\leq 9$ & 17 (56.7) & 4 (13.3) & $9(30)$ & \\
\hline & $(10-12)$ & $153(42.4)$ & $130(36)$ & $78(21.6)$ & \\
\hline \multirow{2}{*}{$\begin{array}{l}\text { Employment status } \\
\text { in the } 1^{\text {st }} \text { trimester }\end{array}$} & No & $178(34.8)$ & 187 (36.6) & $146(28.6)$ & 0.03 \\
\hline & Yes & 231 (31.9) & $233(32.2)$ & 260 (35.9) & \\
\hline \multirow{3}{*}{$\begin{array}{l}\text { Socio-economic } \\
\text { status }\end{array}$} & Medium & 296 (34.1) & 300 (34.6) & $271(31.3)$ & 0.01 \\
\hline & Low & $40(42.1)$ & 32 (33.7) & $23(24.2)$ & \\
\hline & High & $90(28.6)$ & $97(30.8)$ & $128(40.6)$ & \\
\hline \multirow{4}{*}{$\begin{array}{c}\text { Place of residence } \\
\text { [thous. } \\
\text { inhabitants] }\end{array}$} & $>500$ & $153(31.2)$ & $165(33.7)$ & $172(35.1)$ & 0.01 \\
\hline & $<10$ & $115(42.9)$ & 85 (31.7) & $68(25.4)$ & \\
\hline & $10-100$ & 65 (33.5) & $62(32)$ & $67(34.5)$ & \\
\hline & $100-500$ & $102(29)$ & $128(36.4)$ & $122(34.7)$ & \\
\hline \multirow{3}{*}{$\begin{array}{c}\text { Pre-pregnancy BMI } \\
{\left[\mathrm{kg} / \mathrm{m}^{2}\right]}\end{array}$} & $(18.5-24.99)$ & $327(35)$ & $298(31.9)$ & $308(33)$ & 0.001 \\
\hline & $<18.5$ & $47(41.2)$ & 40 (35.1) & $27(23.7)$ & \\
\hline & $\geq 25$ & $54(22.6)$ & $96(40.2)$ & 89 (37.2) & \\
\hline \multirow{3}{*}{$\begin{array}{c}\text { Gestational weight } \\
\text { gain }\end{array}$} & Recommended & $136(32)$ & 145 (34.1) & 144 (33.9) & 0.3 \\
\hline & Low & 177 (31.9) & $194(35)$ & $183(33)$ & \\
\hline & High & $88(39.3)$ & $74(33)$ & $62(27.7)$ & \\
\hline \multirow{2}{*}{\begin{tabular}{|c|}
$\begin{array}{c}\text { Pregnancy } \\
\text { complications }\end{array}$ \\
\end{tabular}} & No & $178(33)$ & $181(33.6)$ & $180(33.4)$ & 0.9 \\
\hline & Yes & 251 (33.4) & 254 (33.8) & $246(32.8)$ & \\
\hline \multirow{2}{*}{$\begin{array}{c}\text { Cotinine level in 1st } \\
\text { tromester }\end{array}$} & $\leq 10 \mathrm{ng} / \mathrm{ml}$ & $317(32.8)$ & 332 (34.4) & $317(32.8)$ & 0.02 \\
\hline & $>10 \mathrm{ng} / \mathrm{ml}$ & $51(45.5)$ & $36(32.1)$ & $25(22.3)$ & \\
\hline \multirow{2}{*}{$\begin{array}{c}\text { Alcohol } \\
\text { consumption in } \\
\text { pregnancy }\end{array}$} & No & 397 (32.9) & $407(33.7)$ & $403(33.4)$ & 0.6 \\
\hline & Yes & 29 (34.9) & $31(37.3)$ & $23(27.7)$ & \\
\hline \multirow{2}{*}{ Child gender } & Male & $178(31.2)$ & $212(37.1)$ & $181(31.7)$ & 0.1 \\
\hline & Female & $192(34.3)$ & $174(31.1)$ & $193(34.5)$ & \\
\hline
\end{tabular}

\section{Conclusions}

Diet is a complex exposure variable, which calls for multiple approaches to examine the relationship between diet and disease risk. Dietary pattern analysis is one of these approaches. The study results highlight the need for educational programs and interventions focused on healthy diet quality during pregnancy. 